\title{
A REMARK ON PARTIAL LINEAR SPACES OF GIRTH 5 WITH AN APPLICATION TO STRONGLY REGULAR GRAPHS
}

\author{
A. E. BROUWER and A. NEUMAIER
}

Received April 10, 1984

We derive a lower bound on the number of points of a partial linear space of girth 5 . As an application, certain strongly regular graphs with $\mu=2$ are ruled out by observing that the first subconstituents are partial linear spaces.

\section{Partial linear spaces of girth 5}

A partial linear space consists of a set of points and a set of lines (subsets of the point set) such that any two lines have at most one point in common. Collinear points are called adjacent or neighbours. The girth of a partial linear space is the length of a shortest circuit.

In view of the application to strongly regular graphs we shall use $k$ for the number of points and $\lambda$ for the valency (of the pointgraph) of a partial linear space.

Theorem. A connected partial linear space with girth at least 5 and more than one line (lines possibly of varying size) in which every point has $\lambda$ neighbours, contains $k \geqq$ $\geqq \lambda(\lambda+3) / 2$ points.

Proof. Let $L$ be a line of size $l$. Denote by $T$ the set of points at distance at least two from $L$. Then $|T|=k-l(\lambda+2-l)$, and $l \leqq \lambda$ since a line of size $\lambda+1$ would be a component. Let $x_{i}$ be the number of points in $T$ having exactly $i$ neighbours at distance one from $L$. We have

(i) $\sum_{i} x_{i}=k-l(\lambda+2-l)$,

(ii) $\quad \sum_{i} i x_{i} \geqq l(\lambda+1-l)(l-1)$,

(iii) $\sum_{i}\left(\begin{array}{l}i \\ 2\end{array}\right) x_{i} \leqq\left(\begin{array}{l}l \\ 2\end{array}\right)(\lambda+1-l)^{2}$.

Hence

$$
\begin{aligned}
& 0 \leqq \sum_{i}(i-(\lambda+2-l))^{2} x_{i}=2 \sum_{i}\left(\begin{array}{l}
i \\
2
\end{array}\right) x_{i}-(2 \lambda+3-2 l) \sum_{i} i x_{i}+(\lambda+2-l)^{2} \sum_{i} x_{i} \leqq \\
& \leqq l(l-1)(\lambda+1-l)^{2}-l(l-1)(\lambda+1-l)(2 \lambda+3-2 l)+(\lambda+2-l)^{2}(k-l(\lambda+2-l)),
\end{aligned}
$$


whence

$$
k(\lambda+2-l)^{2} \geqq l(\lambda+2-l)((\lambda+2)(\lambda+1-l)+1)
$$

which can be written as

$$
k \geqq \frac{1}{2} \lambda(\lambda+3)+\frac{(\lambda+2)(\lambda-l)(2 l-3-\lambda)}{2(\lambda+2-l)}
$$

It follows that if there is a line of size $l$ with $l \geqq(\lambda+3) / 2$ then $k \geqq \lambda(\lambda+3) / 2$ (with strict inequality unless $l=\lambda$ or $l=(\lambda+3) / 2$ ).

If $l$ is relatively small then we can improve on estimate (ii). Let $m$ be the size of the longest line intersecting $L$. We have

(ii) $\sum_{i} i x_{i} \geqq l(\lambda+1-l)(\lambda+1-m)$.

Hence, evaluating $0 \leqq \sum_{i}(i-l)(i-l-1) x_{i}$ we find

$$
k \geqq(\lambda+1)^{2}-l \lambda-\frac{2 l(m-l)(\lambda+1-l)}{l+1}
$$

It follows that if the longest line in our partial linear space has length at most $(\lambda+1) / 2$ (putting $m \leqq l \leqq(\lambda+1) / 2)$ then $k \geqq(\lambda+1)(\lambda+2) / 2=\lambda(\lambda+3) / 2+1$.

In case the longest line has length $(\lambda+2) / 2$, we have to estimate somewhat more carefully. If there is a line $L$ of length $l \leqq \lambda / 2$ such that each line intersecting $L$ has length at most $\lambda / 2$ then $k \geqq \lambda^{2} / 2+2 \lambda+1$. This shows that for smaller $k$ there are many lines of size $\lambda / 2+1$; in fact too many.

Write $|M|=1+s_{M}$ for each line $M$. Considering the lines $M$ distinct from $L$ passing through a point $x \in L$ we see that $x$ is at distance two from $\sum s_{M}\left(\lambda-s_{M}\right)$ points in $T$. But $\sum s_{M}=\lambda+1-l$, so $\sum s_{M}\left(\lambda-s_{M}\right)=\lambda(\lambda+1-l)-(\lambda+1-l)^{2}+$ $+\sum_{M \neq N} s_{M} s_{N} \geqq(l-1)(\lambda+1-l)+\left(n_{x}-1\right)\left(2(\lambda+1-l)-n_{x}\right)$ where $n_{x}=\sum_{M} 1$ is the number of lines intersecting $L$ in the point $x$. of $L$. Then

Let $n_{x}=1$ for $j$ points of $L$, so that $n_{x} \geqq 2$ for the remaining $l-j$ points

(ii) $^{\prime \prime} \quad \sum i x_{i} \geqq l(l-1)(\lambda+1-l)+2(l-j)(\lambda-l)$.

In particular, for $l=\lambda / 2+1$ we find, evaluating $0 \leqq \sum(i-l)(i-l+1) x_{i}$, that

$$
k \geqq \frac{1}{2} \lambda^{2}+\lambda+1+\frac{4(l-2)(l-j)}{l}
$$

On the other hand, if for some $j \in \mathbf{N}$ each line of size $l=\lambda / 2+1$ intersects at least $j+1$ others of this size then considering $j(j+1)$ lines of size $l$ intersecting such lines intersecting a given line $L$ we find $|T|=k-l^{2} \geqq j(j+1)(l-1) / 2=\lambda j(j+1) / 4$.

This shows that if the linear space contains lines of size $l=\lambda / 2+1$ then

(*) $\quad k \geqq \max _{0 \leqq j \geqq l} \min \left\{\frac{1}{2} \lambda^{2}+3 \lambda-3-j \frac{4 l-8}{l}, \frac{1}{4} \lambda j(j+1)+\frac{1}{4} \lambda^{2}+\lambda+1\right\}$. 
Hence, for $\lambda=2,4,6,8,10$ we find $k \geqq 5,15,27,46,67$ respectively, and in general putting $j=\lceil\sqrt{\lambda}\rceil$ we find $k>\lambda(\lambda+3) / 2$ for $\lambda>6$. This proves the theorem.

Remark. Equality holds in the theorem iff $\lambda=2$ and $k=5$. (This partial linear space exists - it is a pentagon.) For: if there is a line of size $\lambda / 2+1$ then $k>\lambda(\lambda+3) / 2$ unless $\lambda=2$ or $\lambda=6$. But if $\lambda=6$ and $k=27$ one sees that each line of size 4 intersects exactly three others and that each point in $T$ has three or four neighbours at distance one of $L$ (where $l=4$ ) - hence lines of size 4 do not intersect in $T$ and $|T| \geqq 18, k \geqq 34$, contradiction.

Hence there are no lines of size $\lambda / 2+1$, but each line of size at most $(\lambda+1) / 2$ intersects a longer line, so there are lines of size $(\lambda+3) / 2$ or $\lambda$. In the former case $(l=(\lambda+3) / 2)$ we may suppose $\lambda>3$. We see that $j=l$, i.e., each line of size $(\lambda+3) / 2$ intersects only lines of size $(\lambda+1) / 2$. Let there be $a$ lines of size $(\lambda+3) / 2$. Then there are $(\lambda-a)(\lambda+3) / 2$ points not in one of these lines, and $a(\lambda-1)(\lambda+3) / 4$ incidences of such points with $(\lambda+1) / 2$-lines. But each point is in at most two $(\lambda+1) / 2$ lines, so $(\lambda-a)(\lambda+3) \geqq a(\lambda-1)(\lambda+3) / 4$ and $a \leqq 3$. If $a \geqq 1$ then we find a line $l$ with $l=(\lambda+1) / 2$ intersecting only one line of size $(\lambda+3) / 2$, i.e., with $j=1$. From $0 \leqq \sum(i-l-1)^{2} x_{i}$ one finds $l \leqq 1$, contradiction.

Hence there are no lines of size $(\lambda+3) / 2$ and all lines have size 2 or $\lambda$.

If some point is only in lines of size 2 then it has $\lambda$ neighbours and $\lambda(\lambda-1)$ Doints at distance 2 so that $k \geqq \lambda^{2}+1$. On the other hand, if $\lambda>2$ and each point is in a line of size $\lambda$, then let $L$ be a line of size $\lambda$. Each of its $\lambda$ neighbours is in a line of length $\lambda$, and these lines cannot intersect, so $|T| \geqq \lambda(\lambda-1)$ and $k \geqq \lambda(\lambda+1)$. This proves our claim.

Remark. We do not know the right order of magnitude of the lower bound. The Theorem gives something of order $\lambda^{2} / 2$ - on the other hand, the Moore graphs of diameter two are examples with $k=\lambda^{2}+1$. For small $\lambda$ we have:

$$
\begin{array}{ll}
\lambda=2, & k=5, \quad \text { the pentagon. } \\
\lambda=3, & k=10, \quad \text { the Petersen graph. } \\
\lambda=4, & k=15:
\end{array}
$$

There is a unique partial linear space on 15 points with 10 lines of size 3 and girth 5 . Its point-graph is distance regular with parameters $i(4,2,1 ; 1,1,4)$. It is obtained from the generalised quadrangle $G Q(2,2)$ by deleting a parallel class of lines. It is the line graph of the Petersen graph.

Now these three examples are regular: all lines have the same size. But for regular partial linear spaces these same methods yield stronger bounds: we have $k \geqq \lambda(\lambda-l+2)+1$ if all lines have size $l$. If $\lambda \leqq l(l-1)$ this can be strengthened to

$$
k \geqq l^{2}(\lambda-2 l+3)+\frac{l(l-1)^{3}}{\lambda} .
$$

(In case $\lambda=l(l-1)$, equality would mean that we have a strongly regular graph with $\mu=1$ and discriminant $(l-1) \sqrt{5}$, hence equality occurs only for $l=2$. In general equality means that we have a strongly regular graph with $\mu=1$ in the first 
case and a distance regular graph of diameter at most three in the second case the linegraph of a system satisfying one bound with equality, satisfies the other with equality. This yields very strong conditions on the parameters, and only finitely many examples are known.)

An infinite family of regular examples is provided by the incidence graphs of finite projective planes: they have $k=2\left(\lambda^{2}-\lambda+1\right)$, for $\lambda=q+1, q$ a primepower. $k=10$ namely

Concerning irregular examples, there are precisely two others with $\lambda=3$,

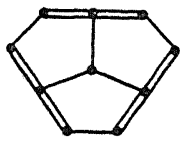

and

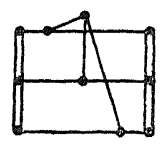

For $\lambda=2, k=5$ and $\lambda=4, k=15$ there are no irregular examples. There are no examples with $\lambda=5, k=21$.

\section{Strongly regular graphs with $\mu=2$}

Let $G$ be a graph such that two nonadjacent vertices have at most two common neighbours. Let $x$ be a fixed vertex, and $H=\Gamma(x)$ the graph induced on the neighbours of $x$. Then each edge of $H$ is contained in a unique maximal clique, and points and maximal cliques of $H$ form a partial linear space of girth at least five.

Now if $G$ is moreover regular of valency $k$ and each edge is in exactly $\lambda$ triangles then by the Theorem, either $k \geqq \lambda(\lambda+3) / 2$ or $\Gamma(x)$ is a disjoint union of lines of size $\lambda+1$. It follows that $(\lambda+1) \mid k$ and that $G$ itself is a partial linear space.

In particular, this holds for strongly regular graphs with parameters $v, k, \lambda, \mu$ (as defined, e.g., in [2]) where $\mu=2$. Thus:

Corollary. A strongly regular graph with $\mu=2$ and $k<\lambda(\lambda+3) / 2$ is a partial quadrangle; in particular it satisfies the divisibility condition $(\lambda+1) \mid k$.

This Corollary rules out infinitely many feasible sets of parameters of strongly regular graphs that just escape the claw bound ([2], Theorem 4.7 (iii)). For example, if $\mu=2$ and the smallest eigenvalue is -4 then its multiplicity is $24 \lambda+210 /(\lambda+6)$ so that $\lambda \in\{0,1,4,8,9,15,24,29,36,64,99,204\}$. The claw bound rules out the parameter sets with $\lambda \geqq 24$. The Corollary implies that also $\lambda \in\{8,9,15\}$ is impossible, thus leaving the parameter sets $(v, k, \lambda)=(56,10,0),(99,14,1),(300,26,4)$. (The first one corresponds to the Gewirtz graph [1], the other two are unknown.)

An infinite series of ruled out parameter sets is e.g. $(v, k, \lambda)=$ $=\left(t^{2}(6 t+11)(3 t+2), 6 t^{2}+10 t-2,5 t-2\right)$, admissible for $t \equiv 1,2,4$ or $6(\bmod 7)$ but excluded by the corollary for $t \geqq 2$.

Looking at the table we found one parameter set that just escaped the bound, namely $(v, k, \lambda)=(1944,67,10)$. But also this one is easily ruled out. Returning to the proof of the theorem in the special case $k=67, \lambda=10$ we see that there are no lines of size 7,8 or 9 and that each line of length 6 intersects at least 4 and hence exactly 4 other lines of size 6 . If there is a line of size 6 then there are at least $1+4+$ $+12=17$ such lines, together containing at least $(2+4 / 2) \cdot 17=68$ points, a
contradiction. 

tradiction.

Hence there are only lines of size 2 or 10 and $k \geqq \lambda^{2}+1=101$, again a con-

Clearly our result can also be applied to other distance regular graphs, but we have no examples at present.

Table of "feasible" parameters for strongly regular graphs with $v \leqq 2000$ and $\mu=2$ but not with the parameters of a net

\begin{tabular}{rrrrrrrl}
\hline \multicolumn{1}{c}{$v$} & \multicolumn{1}{c}{$k$} & \multicolumn{1}{c}{$\lambda$} & \multicolumn{1}{c}{$r$} & \multicolumn{1}{c}{$s$} & \multicolumn{1}{c}{$f$} & \multicolumn{1}{c}{ existence } \\
\hline 4 & 2 & 0 & 0 & -2 & 2 & 1 & 4-cycle (unique) \\
16 & 5 & 0 & 1 & -3 & 10 & 5 & Clebsch graph (unique) \\
56 & 10 & 0 & 2 & -4 & 35 & 20 & Gewirtz graph (unique) \\
85 & 14 & 3 & 4 & -3 & 34 & 50 & $?$ \\
99 & 14 & 1 & 3 & -4 & 54 & 44 & $?$ \\
243 & 22 & 1 & 4 & -5 & 132 & 110 & Berlekamp-Seidel-van Lint graph \\
300 & 26 & 4 & 6 & -4 & 117 & 182 & $?$ \\
352 & 26 & 0 & 4 & -6 & 208 & 143 & $?$ \\
456 & 35 & 10 & 11 & -3 & 95 & 360 & Ruled out by the claw bound \\
630 & 37 & 4 & 7 & -5 & 259 & 370 & $?$ \\
704 & 37 & 0 & 5 & -7 & 407 & 296 & $?$ \\
736 & 42 & 8 & 10 & -4 & 207 & 528 & Ruled out in this note \\
875 & 46 & 9 & 11 & -4 & 230 & 644 & Ruled out in this note \\
1176 & 50 & 4 & 8 & -6 & 500 & 675 & $?$ \\
1276 & 50 & 0 & 6 & -8 & 725 & 550 & $?$ \\
1625 & 58 & 3 & 8 & -7 & 754 & 870 & $?$ \\
1944 & 67 & 10 & 13 & -5 & 536 & 1407 & Ruled out in this note \\
1961 & 70 & 15 & 17 & -4 & 370 & 1590 & Ruled out in this note
\end{tabular}

(Here $k, r, s$ are the eigenvalues of the graph, with multiplicities $1, f, g$.)

\section{References}

[1] A. Gewlrtz, The uniqueness of $g(2,2,10,56)$, Trans. New York Acad. Sci., 31 (1969), 656-675.

[2] A. NeumaIER, Strongly regular graphs with smallest eigenvalue $-M$, Archiv der Mathematik, 33 (1979), 392-400.

\section{A. E. Brouwer}

Mathematical Centre

Kruislaan 413, Amsterdam

The Netherlands

\section{A. Neumaier}

Institut für Angewandte Mathematik

Universität Freiburg,

D-7800 Freiburg, BRD 